Marc Pillarz*, Axel von Freyberg, Dirk Stöbener und Andreas Fischer

\title{
Optische Messung zahnindividueller Formparameter am Beispiel von Geradverzahnungen
}

\author{
Optical measurement of tooth-individual shape parameters of spur gears
}

DOI 10.1515/teme-2021-0068

Zusammenfassung: Der Bedarf an skalierbaren Messsystemen für die umfassende Qualitätsprüfung zahnindividueller Formparameter metergroßer Zahnräder mit einstelliger Unsicherheit im Mikrometerbereich wächst. Taktile Standardverzahnungsmesssysteme sind präzise, aber aufgrund der limitierten Geschwindigkeit und des begrenzten Messvolumens für eine umfassende Qualitätsprüfung aller Zähne nur bedingt geeignet. Aus diesem Grund wird ein schnellerer optischer Multi-Distanz-Messansatz mit modellbasierter Auswertung zur Verzahnungsmessung untersucht. Über bisherige Arbeiten hinausgehend, in denen zunächst der mittlere Grundkreisradius als grundlegender Formparameter von allen Zähnen bestimmt wurde, wird hier die Eignung des Messansatzes zur Erfassung zahnindividueller Formparameter untersucht. Messungen an kleinen Verzahnungen zeigen, dass der zahnindividuelle Grundkreisradius im Mittel mit einer Unsicherheit $<10 \mu \mathrm{m}(k=2)$ bestimmt werden kann, was eine vielversprechende Grundlage für die zukünftige Anwendung des Multi-Distanz-Messansatzes bei Großverzahnungen bietet.

Schlüsselwörter: optische Verzahnungsmessung, MultiDistanz-Messungen, modellbasierte Auswertung, konfokalchromatischer Sensor, Messunsicherheit.

Abstract: The need for scalable measuring systems for comprehensive quality inspection of tooth-specific form parameters of meter-sized gears with single-digit uncertainty in the micrometer range is growing. Tactile standard gear

\footnotetext{
*Korrespondenzautor: Marc Pillarz, Universität Bremen, Bremer Institut für Messtechnik, Automatisierung und Qualitätswissenschaft (BIMAQ), E-Mail: m.pillarz@bimaq.de Axel von Freyberg, Universität Bremen, Bremer Institut für Messtechnik, Automatisierung und Qualitätswissenschaft (BIMAQ), E-Mail: a.freyberg@bimaq.de

Dirk Stöbener, Andreas Fischer, Universität Bremen, Bremer Institut für Messtechnik, Automatisierung und Qualitätswissenschaft (BIMAQ), Universität Bremen, MAPEX Center for Materials and Process, E-Mail: d.stoebener@bimaq.de, andreas.fischer@bimaq.de
}

measuring systems are precise, but due to their limited speed and measuring volume, they are only suitable to a limited extent for comprehensive quality inspection of all teeth. For this reason, a faster optical multi-distance measurement approach with model-based evaluation is being investigated for gear measurement. Going beyond previous work, where the mean base circle radius was determined as the fundamental shape parameter of all teeth, the suitability of the measurement approach for inspecting tooth-individual shape parameters is investigated here. Measurements on small gears show that the tooth-individual base circle radius can be determined on average with an uncertainty $<10 \mu \mathrm{m}(k=2)$, which provides a promising basis for the future application of the multi-distance measurement approach on large gears.

Keywords: optical gear measurement, multi-distance measurements, model-based evaluation, confocal chromatic sensor, measurement uncertainty.

\section{Einleitung}

\subsection{Motivation}

Für eine gleichmäßige Kraftübertragung in Getrieben ist die Zahnflankenform entscheidend. Der Grundkreisradius einer Stirnverzahnung mit Evolventenprofil ist der formgebende Parameter für die Geometrie der Zahnflanke. Üblicherweise wird anstelle des Grundkreisradius die Profilwinkelabweichung zur Bewertung der Zahnflanke ausgewertet, jedoch sind bei Stirnverzahnungen beide Größen ineinander umrechenbar. Abweichungen des Grundkreisradius von der Nenngeometrie führen zu einem ungleichmäßigen Übertragungsverhalten und vorzeitigem Verschleiß der Verzahnungen. Die Herstellung von Verzahnungen ist daher mit engen Toleranzen im Mikrometerbereich verbunden [4]. Abgeleitet aus [4] werden je nach Verzahnungsqualität Grundkreisradiusabweichungen im niedrigen zweistelligen Mikrometerbereich toleriert. Um die Zuverlässigkeit von 
Getrieben zu steigern, muss daher der Grundkreisradius mindestens mit einer Messunsicherheit im einstelligen Mikrometerbereich $(<10 \mu \mathrm{m})$ erfasst werden.

Bei Großverzahnungen müssen zudem die Daten aller Zähne erfasst und individuell bewertet werden, da die Herstellung von Großverzahnungen in der Regel auf diskontinuierlichen Verfahren basiert. Darüber hinaus stellen geometrische Messungen an Großverzahnungen wegen der metergroßen Abmessungen und Massen größer 500 kg eine zusätzliche logistische Herausforderung dar. Deshalb sind zur Großverzahnungsmessung Messsysteme mit einem flexiblen und variablen Messvolumen erforderlich. Zusammenfassend bedarf es skalierbarer Verzahnungsmesssysteme, die die Daten aller Zähne erfassen, um zahnindividuelle Formparameter wie den Grundkreisradius von Verzahnungen mit einer Unsicherheit im einstelligen Mikrometerbereich zu quantifizieren.

\subsection{Stand der Technik}

Standardmesssysteme für Verzahnungen, wie taktile Koordinatenmessgeräte (KMG) und Verzahnungsmessgeräte $[1,5,10]$, sind präzise, aber hinsichtlich der Geschwindigkeit und des Messvolumens limitiert. Aufgrund der limitierten Geschwindigkeit werden in Standardverzahnungsprüfungen typischerweise nur vier über den Umfang des Zahnrads verteilte Zähne gemessen [1]. Dieser stichprobenartige Prüfumfang erlaubt jedoch keine zuverlässige Qualitätsprüfung aller Zähne, weshalb die taktilen Standardmesssysteme nur bedingt für umfangreiche Großverzahnungsmessungen geeignet sind.

In jüngster Zeit werden daher schnellere optische Verzahnungsmesskonzepte untersucht, deren Potential zunächst am Beispiel kleiner Verzahnungen aufgezeigt wird. Chen et al. detektieren die 3D-Topologie der Zahnflanken eines Stirnrads mittels Moiré-Projektion und ermitteln zahnindividuelle Formparameter wie beispielsweise die Profilwinkelabweichung [2]. Dabei wird eine Messunsicherheit $<5 \mu \mathrm{m}$ (ein Erweiterungsfaktor ist nicht angegeben) erreicht. Die erzielten Messergebnisse zeigen ein großes Potenzial für Verzahnungsmessungen, eine Anwendung für Großverzahnungen bzw. die Skalierbarkeit des Ansatzes wird jedoch nicht diskutiert. Ein weiterer optischer Verzahnungsmessansatz wird in [8] beschrieben und basiert auf Messungen mit einem Laserlinien-Triangulationssensor in Kombination mit einem Drehtisch. Guo et al. zeigen, dass die Gesamtprofilabweichung der individuellen Zahnflanken mit einer Unsicherheit von $<5 \mu \mathrm{m}$ (ein Erweiterungsfaktor ist nicht angegeben) gemessen werden kann [8]. Skalierbare Messungen von Großverzahnungen wer- den jedoch nicht erwähnt. In [11] stellen Pillarz et al. einen skalierbaren Multi-Distanz-Messansatz zur schnellen Prüfung von Großverzahnungen vor, der auf einen konfokal-chromatischen Abstandssensor in Kombination mit einem Drehtisch basiert. Die optisch gemessene Punktwolke wird hierbei zur Auswertung an ein parametrisches Geometriemodell der Verzahnung approximiert. Mit dem Multi-Distanz-Messansatz lässt sich der mittlere Grundkreisradius von allen Zähnen mit einer Unsicherheit von $<10 \mu \mathrm{m}(k=2)$ bestimmen. Das grundsätzliche Potential dieses Messansatzes zur Erfassung zahnindividueller Formparameter wird im Ausblick erwähnt, eine Untersuchung zur erreichbaren Messunsicherheit ist jedoch ausstehend.

\subsection{Ziel und Struktur des Artikels}

In diesem Artikel wird daher der skalierbare Multi-DistanzMessansatz zur Verzahnungsmessung für die Erfassung zahnindividueller Formparameter mit einer Unsicherheit $<10 \mu \mathrm{m}(k=2)$ am Beispiel des Grundkreisradius untersucht. Dafür wird die modellbasierte Auswertung der Messpunkte um eine weitere Approximationsstufe ergänzt. Das entsprechend erweiterte Messkonzept wird durch den Einsatz eines konfokal-chromatischen Sensors in Kombination mit einem Drehtisch realisiert, um kontinuierliche Abstandsmessungen zu allen Zahnflanken, zunächst an kleinen Verzahnungen, durchzuführen. Der Messansatz wird in Abschnitt 2 beschrieben. Abschnitt 3 erläutert den experimentellen Aufbau zu den Multi-DistanzVerzahnungsmessungen. Die Messergebnisse werden anschließend in Abschnitt 4 veranschaulicht und mit taktilen Referenzmessungen basierend auf einer Standardverzahnungsauswertung validiert. Der Artikel schließt mit einer Zusammenfassung in Abschnitt 5.

\section{Messprinzip}

\subsection{Messgröße Grundkreisradius}

Zur umfangreichen und aussagekräftigen Qualitätsbeurteilung von Verzahnungen sind zahnindividuelle Formparameter zu bewerten. Der Grundkreisradius ist der formgebende Parameter für die Geometrie der Zahnflanken und wird in diesem Artikel als grundlegender Formparameter betrachtet. Typischerweise wird bei der Standardverzahnungsmessung die Profilwinkelabweichung zur Bewertung der Zahnflanke herangezogen und nicht der Grundkreisradius. Der Ist-Grundkreisradius korreliert jedoch mit der 
Profilwinkelabweichung und kann gemäß der Beziehung

$$
r_{\mathrm{b}}=r_{\mathrm{b}, \mathrm{n}} \cdot \frac{f_{\mathrm{H} \alpha}}{L_{\alpha}}+r_{\mathrm{b}, \mathrm{n}}
$$

aus dem Nenngrundkreisradius $r_{\mathrm{b}, \mathrm{n}}$, der Profilwinkelabweichung $f_{\mathrm{H} \alpha}$ und dem Auswertebereich $L_{\alpha}$ berechnet werden. Die Profilwinkelabweichung definiert die Abweichung der tatsächlichen Winkellage im Vergleich zur Soll-Winkellage der Zahnflanke [9]. Zur Abschätzung der erreichbaren Messunsicherheit bei der Messung zahnindividueller Grunkreisradien mittels des Multi-Distanz-Messansatzes wird aus taktil gemessenen, klassisch ausgewerteten Profilwinkelabweichungen und durch Anwendung von Gleichung (1) ein Referenzwert für den Grundkreisradius ermittelt.

\subsection{Modellbasierte Auswertung des Grundkreisradius}

In $[11,12]$ stellen Pillarz et al. ein modellbasiertes Auswerteverfahren zur Berechnung des Formparameters mittleren Grundkreisradius auf Basis eines Geometriemodells von Evolventenverzahnungen nach $[6,7]$ vor. Ein Punkt $P_{\mathrm{i}}$ auf einer idealen Zahnflanke

$$
\begin{aligned}
P_{\mathrm{i}} & =\left[\begin{array}{c}
x_{\mathrm{i}} \\
y_{\mathrm{i}}
\end{array}\right]=r_{\mathrm{b}} \cdot\left[\begin{array}{c}
\cos \left(\xi_{\mathrm{i}}+\theta_{\mathrm{z}}-\psi_{\mathrm{b}}+\phi_{0}\right) \\
\sin \left(\xi_{\mathrm{i}}+\theta_{\mathrm{z}}-\psi_{\mathrm{b}}+\phi_{0}\right)
\end{array}\right] \\
& +r_{\mathrm{b}} \cdot \xi_{\mathrm{i}} \cdot\left[\begin{array}{c}
\sin \left(\xi_{\mathrm{i}}+\theta_{\mathrm{z}}-\psi_{\mathrm{b}}+\phi_{0}\right) \\
-\cos \left(\xi_{\mathrm{i}}+\theta_{\mathrm{z}}-\psi_{\mathrm{b}}+\phi_{0}\right)
\end{array}\right]+\left[\begin{array}{c}
x_{\mathrm{t}} \\
y_{\mathrm{t}}
\end{array}\right]
\end{aligned}
$$

kann durch die Lageparameter $\left(\xi_{\mathrm{i}}\right.$ : Wälzwinkel, $\theta_{\mathrm{z}}$ : Lage der Mittelachse von Zahn $z, \psi_{\mathrm{b}}$ : Zahndickenhalbwinkel, $\phi_{0}$ : Verdrehung zum Werkstückkoordinatensystem und $\left(x_{\mathrm{t}}, y_{\mathrm{t}}\right)$ : Translation zum Werkstückkoordinatensystem und Mittelpunkt der Verzahnung) und dem Formparameter Grundkreisradius $r_{\mathrm{b}}$ in einem Messkoordinatensystem beschrieben werden. Die Berechnung des Grundkreisradius erfolgt daraufhin mit der Lösung des inversen Problems von Gleichung (2), wobei die fünf $(i+4)$ Parameter $\left(\xi_{\mathrm{i}}, \phi_{0}, x_{\mathrm{t}}, y_{\mathrm{t}}, r_{\mathrm{b}}\right)$ unbekannt sind und mit nur einem Messpunkt $P_{\mathrm{i}}=\left(x_{\mathrm{i}}, y_{\mathrm{i}}\right)$ ein unterbestimmtes Gleichungssystem vorliegt. Um das inverse Problem zu lösen, sind dementsprechend mindestens vier Messpunkte $(i=1 \ldots 4)$ erforderlich.

Die gemessenen Zahnflanken weichen zudem von der Nenngeometrie ab. Zur Bestimmung des tatsächlichen Grundkreisradius wird daher ein iterativer Ansatz verwendet. Eine mittlere ideale Evolvente wird unter Berücksichtigung der Abweichungen (Lotabstände $d_{\mathrm{plu}, \mathrm{i}}$ ) zwischen den gemessenen Zahnflanken und der idealen Zahnflanke in die Messpunkte aller gemessenen Zahnflanken approximiert. Die Berechnung der Lotabstände erfolgt auf Basis von [13]. Durch Minimierung der Summe der quadrierten Lotabstände zu den gemessenen Punkten

$$
\min _{\xi_{\mathrm{i}}, \phi_{0}, x_{\mathrm{t}}, y_{\mathrm{t}}, r_{\mathrm{b}}}\left(\sum_{i=1}^{k} d_{\mathrm{plu}, \mathrm{i}}^{2}\right)
$$

wird nach der Methode der kleinsten Quadrate eine ideale Evolvente in Abhängigkeit von den Parametern $\left(\xi_{\mathrm{i}}, \phi_{0}\right.$, $\left.x_{\mathrm{t}}, y_{\mathrm{t}}, r_{\mathrm{b}}\right)$, d.h. einschließlich des gesuchten mittleren Grundkreisradius $r_{\mathrm{b}}$ berechnet.

Die Approximation einer Evolvente zur Berechnung des Grundkreisradius ist ein komplexes nichtlineares Minimierungsproblem mit einem multidimensionalen Lösungsraum. Bei der zahnindividuellen Grundkreisradiusapproximation erhöhen die Randbedingungen einer unvollständigen Erfassung der Evolvente und insbesondere die lokale Häufung der Messpunkte zusätzlich die Wahrscheinlichkeit, nicht das globale Minimum zu finden. Systematische Abweichungen in der Grundkreisradiusberechnung sind die Folge. Zur Reduzierung dieser systematischen Abweichungen wird eine zweistufige Approximation durchgeführt, wodurch der Lösungsraum schrittweise bestimmt wird. Zunächst werden die freien Parameter $\left(\xi_{\mathrm{i}}, \phi_{0}, x_{\mathrm{t}}, y_{\mathrm{t}}, r_{\mathrm{b}}\right)$ über alle gemessenen Zähne gemäß Gleichung (3) bestimmt. In einer anschließenden Approximation nach Gleichung (3) werden dann die ermittelten Parameter $\left(x_{\mathrm{t}}, y_{\mathrm{t}}\right)$ gesperrt. Die zweite Approximationsstufe hat somit weniger freie Parameter, wodurch systematische Abweichungen bei der Approximation des zahnindividuellen Grundkreisradius auf Basis der Messdaten von nur einer Evolvente verringert werden.

\subsection{Multi-Distanz-Messansatz}

Um die zahnindividuellen Grundkreisradien zu bestimmen, wird zur Messung der Zahnflankenform ein MultiDistanz-Messansatz bestehend aus $n$ optischen Abstandssensoren in Kombination mit einem Drehtisch vorgestellt (vgl. Abb. 1). Die Sensoren sind über den Umfang verteilt und tangential zum Nenngrundkreis im Stirnschnitt des Zahnrads ausgerichtet.

Die Sensoren mit einem Sensorkoordinatensystem $(x, y)$ und der Drehtisch bilden im Drehzentrum das gemeinsame Messkoordinatensystem $\left(x_{\mathrm{r}}, y_{\mathrm{r}}\right)$. Das Sensorsystem wird mit einem bekannten Zahnrad nach dem Ansatz von [11] kalibriert und justiert, da die genaue Sensorposition und -ausrichtung nicht bekannt sind und eine unbekannte Sensoranordnung zu einer systematischen Messabweichung bei der Berechnung des Grundkreisradius führt. Im Gegensatz zur Kalibrierung hinsichtlich 


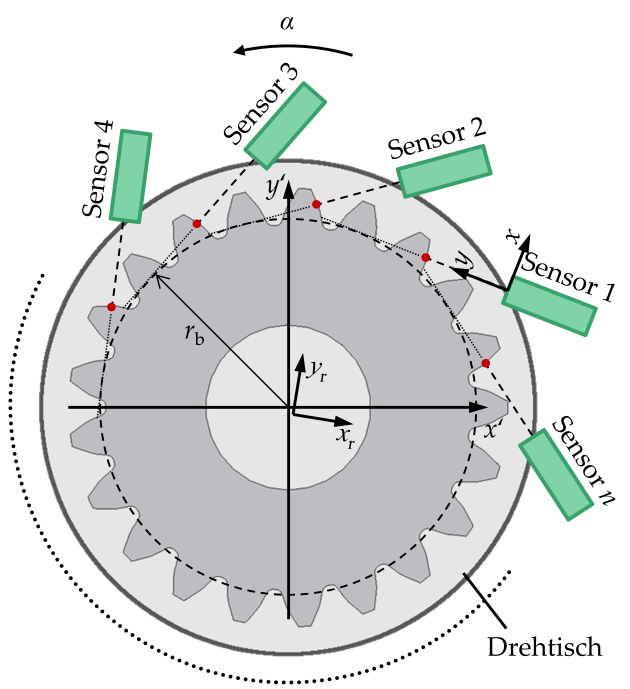

Abb. 1: Modellbasierter Multi-Distanz-Ansatz bestehend aus $n$ optischen Abstandssensoren mit einem Sensorkoordinatensystem $(x, y)$ in Kombination mit einem Drehtisch in einem gemeinsamen Messkoordinatensystem $\left(x_{\mathrm{r}}, y_{\mathrm{r}}\right)$, das kontinuierlich die Zahnform eines Stirnrades mit einem Werkstückkoordinatensystem $\left(x^{\prime}, y^{\prime}\right)$ in Abhängigkeit vom Drehwinkel $\alpha$ misst.

des mittleren Grundkreisradius [13], wird in diesem Artikel das Messsystem zahnindividuell kalibriert. Während das Zahnrad rotiert, werden die Zahnflanken kontinuierlich in Form von Distanzen $d_{\mathrm{i}}$ über die Drehwinkel $\alpha_{\mathrm{i}}$ gemessen. Die scannende Datenerfassung führt zu einem überbestimmten Gleichungssystem, das die iterative Auswertung des Formparameters Grundkreisradius pro Zahn nach Gleichung (3) ermöglicht.

\section{Experimenteller Messaufbau}

\subsection{Messobjekt}

Für die Verzahnungsmessungen wird ein nicht modifiziertes Stirnrad verwendet. Das Zahnrad hat 26 Zähne mit Evolventenprofil, einen Normalmodul von $3,75 \mathrm{~mm}$ und einen Nenngrundkreisradius von 45,8100 $\mathrm{mm}$.

Zur Abschätzung der erreichbaren Messunsicherheit bei der Erfassung zahnindividueller Formparameter mittels dem Multi-Distanz-Messansatz wird eine Referenzmessung mit einem Leitz PMM-F 30.20.7 PortalKoordinatenmessgerät durchgeführt. Bei dieser Standardverzahnungsmessung werden die Profilwinkelabweichungen aller Zähne mit einer geschätzten Unsicherheit von $1 \mu \mathrm{m}$ $(k=1)$ gemessen, und basierend auf Gleichung (1) die
Referenzwerte für die zahnindividuellen Grundkreisradien bestimmt.

Zur Kalibrierung des modellbasierten Multi-DistanzAnsatzes wird ein Kalibrierzahnrad verwendet, welches mittels taktiler Referenzmessungen charakterisiert ist. Da das Kalibrierzahnrad Ähnlichkeitsbedingungen bezüglich des zu messenden Zahnrades erfüllen muss [3], wird ein nicht modifiziertes Stirnrad mit nominell gleicher Geometrie verwendet.

\subsection{Messanordnung}

In diesem Artikel wird die erreichbare Messunsicherheit für zahnindividuelle Grundkreisradien auf Basis eines MultiDistanz-Messansatzes bestehend aus einem Sensor $(n=1)$ in Kombination mit einem Drehtisch abgeschätzt. Der Messaufbau des Multi-Distanz-Messansatzes ist in Abb. 2 dargestellt. Als Rotationseinheit wird der Drehtisch des Portal-Koordinatenmessgeräts Leitz PMM-F 30.20.7 eingesetzt, auf dem das Zahnrad durch eine spezielle Spanneinheit positioniert wird. Zur Messung der Form der Zahnflanken wird ein konfokal-chromatischer Sensor IFS2405-10 von MicroEpsilon eingesetzt. Der Sensor hat einen Messbereich von $10 \mathrm{~mm}$ bei einem Arbeitsabstand von $50 \mathrm{~mm}$ und ist mit einer Abstandsunsicherheit von 2,5 $\mu \mathrm{m}$ spezifiziert $(k=1)$. Der Messfleck hat auf den Zahnoberflächen einen mittleren Durchmesser von $16 \mu \mathrm{m}$. Der Sensor wird so positioniert, dass bei der Erfassung der Zahnflanken der gesamte Messbereich genutzt wird. Um den Sensor tangential zum Nenngrundkreis des Zahnrads auszurichten, wird eine manuelle Dreheinheit verwendet. Durch einen Taumel, der aus einer exzentrischen und ggf. leicht schrägen Einspannung des Zahnrades auf dem Drehtisch resultiert, weicht die reale Sensorausrichtung jedoch von der idealen Ausrichtung ab. Die Kalibrierung des Sensorsystems erfolgt, indem das Kalibrierzahnrad nahezu konzentrisch auf dem Drehtisch mittels der Spanneinheit montiert wird. Eine Herausforderung für den Aufbau ist die reproduzierbare Einspannung des Kalibrierzahnrads und des anschließend zu messenden Zahnrads. Alle Messungen werden in einem klimatisierten Messraum durchgeführt. Die Temperaturänderungen über die Zeit sind kleiner als 0,4 K pro Stunde.

Bei der Multi-Distanz-Messung misst der konfokalchromatische Sensor den Abstand zur Zahnradoberfläche mit einer Messrate von $1 \mathrm{kHz}$, während das Zahnrad rotiert wird. Wiederholungsmessungen werden immer am gleichen Zahn auf der linken Flankenseite gestartet. Pro Zahn werden 1350 Messpunkte entsprechend der optischen Erreichbarkeit erfasst. Aufgrund der Abschattung durch Nachbarzähne wird nur die Hälfte der Evolvente am 


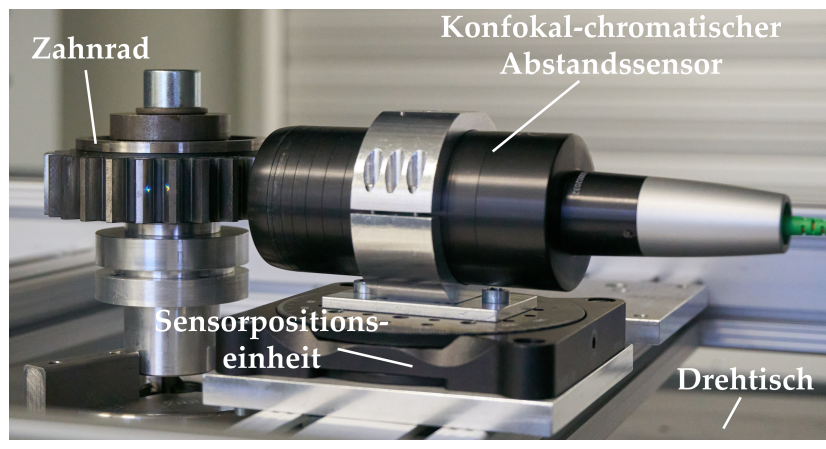

Abb. 2: Messaufbau zur modellbasierten Multi-Distanz-Messung der zahnindividuellen Grundkreisradien eines nicht modifizierten Evolventenstirnrads mit einem Nenngrundkreisradius von 45,8100 mm. Der Multi-Distanz-Ansatz besteht aus einem konfokal-chromatischen Abstandssensor und einem Drehtisch zur Durchführung von scannenden Messungen der Zahnflankenform.

äußeren Ende bis zum Zahnkopf erfasst. Für die modellbasierte Auswertung der zahnindividuellen Grundkreisradien werden die winkelabhängigen gemessenen Abstände anschließend in Koordinaten transformiert.

\section{Verzahnungsmessungen}

Aufgrund der unbekannten Sensoranordnung und der daraus resultierenden systematischen Abweichung bei der Grundkreisradiusbestimmung, muss das Multi-DistanzMesssystem zunächst kalibriert werden. Dazu wird die Geometrie eines bekannten Kalibrierzahnrades gemessen, welche zuvor mittels taktiler Referenzmessungen und auf Basis von Gleichung (1) bestimmt wurde. Die Unsicherheitsabschätzung für die zahnindividuellen Referenzgrundgkreisradien basiert dabei auf einer Fortpflanzungsrechnung mit der geschätzten Unsicherheit für die Profilwinkelabweichung von $1 \mu \mathrm{m}$. Anschließend zu den MultiDistanz-Messungen am Kalibrierzahnrad werden die jeweiligen zahnindividuellen Grundkreisradien modellbasiert ausgewertet. Die Kalibrierung des Systems erfolgt daraufhin in Form einer zahnindividuellen Offsetkorrektur, indem die ermittelten Grundkreisradien mit der zahnindividuellen Referenzgeometrie verglichen werden. Für jeden Zahn ergibt sich so ein Korrekturwert für den Grundkreisradius, welcher auf folgende Multi-Distanz-Messungen an ähnlichen Verzahnungen angewendet werden kann. Die erreichbare Messunsicherheit bei der zahnindividuellen Grundkreisradiusbestimmung wird daraufhin an einem weiteren Stirnrad mit vergleichbarer Nenngeometrie quantifiziert. Das Zahnrad wird entsprechend der Kalibriermessungen auf dem Drehtisch positioniert und eingespannt.
Für die Abschätzung der erreichbaren Messunsicherheit bei der Erfassung zahnindividueller Grundkreisradien werden mit dem Multi-Distanz-Messansatz 22 Wiederholungsmessungen durchgeführt. In Abb. 3 sind die experimentellen Messergebnisse der zahnindividuellen Grundkreisradien veranschaulicht. Die blauen Kreuze symbolisieren die mit dem modellbasierten Multi-DistanzMessansatz bestimmten gemittelten Grundkreisradien für 22 Wiederholungsmessungen pro Zahn, wohingegen die roten Kreise die taktil gemessenen zahnindividuellen Referenzgrundkreisradien darstellen. Darüber hinaus ist die erweiterte Messunsicherheit für $k=2$ angegeben.

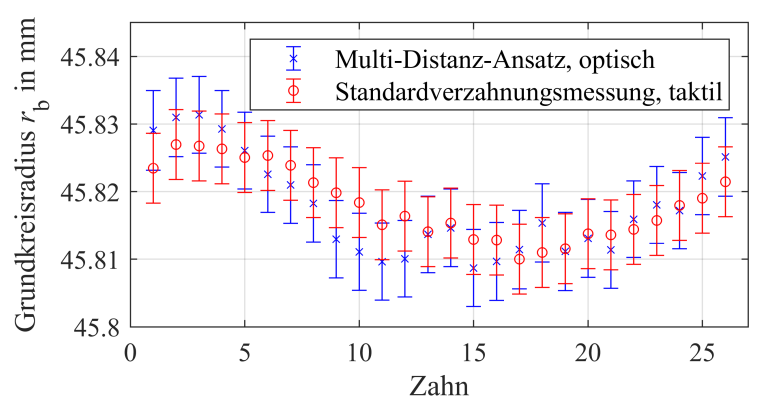

Abb. 3: Experimentelle Ergebnisse der Erfassung der zahnindividuellen Grundkreisradien $r_{\mathrm{b}}$ an einer kleinen Verzahnung. Dargestellt sind die auf Basis des Multi-Distanz-Ansatzes mit einem optischen Sensor erfassten mittleren Grundkreisradien pro Zahn für 22 Wiederholungsmessungen (blaue Kreuze) und die auf Basis einer taktilen Standardverzahnungsmessung ermittelten zahnindividuellen Referenzgrundkreisradien (rote Kreise). Zusätzlich ist die erweiterte Messunsicherheit für $k=2$ für die ermittelten Grundkreisradien angegeben.

Die Messergebnisse in Abb. 3 zeigen, dass die modellbasierten Multi-Distanz-Messungen mit den taktilen Referenzmessungen im Rahmen der erweiterten Messunsicherheiten übereinstimmen. Signifikante systematische Messabweichungen sind mit einer hohen statistischen Sicherheit nicht sichtbar und werden somit zahnindividuell kompensiert. Im Mittel beträgt die erweiterte Messunsicherheit $(k=2)$ des zahnindividuellen Grundkreisradius der Multi-Distanz-Messungen 9,8 $\mu \mathrm{m}$, womit die Zielsetzung von einer Messunsicherheit $<10 \mu \mathrm{m}(k=2)$ knapp erreicht ist. Die erreichbare Messunsicherheit ergibt sich aus zufälligen Messabweichungen aus den optischen DistanzMessungen und der Unsicherheit aus der Kalibrierung des Messsystems. Der Beitrag der zufälligen Messabweichungen resultierend aus der optischen Messung errechnet sich aus der Standardabweichung der 22 Wiederholungsmessungen pro Zahn zu 4,1 $\mu \mathrm{m}(k=1)$ und dominiert die erreichbare Messunsicherheit. Durch eine Steigerung der 
Messpunktezahl kann der Beitrag der zufälligen Messabweichungen resultierend aus der optischen Messung und damit die gesamte erreichbare Messunsicherheit bei der zahnindividuellen Grundkreisradiusbestimmung mittels des modellbasierten Multi-Distanz-Messansatzes zukünftig noch reduziert werden. Daraufhin ist dann die Limitierung der erreichbaren Messunsicherheit aufgrund der Kalibrierunsicherheit $\mathrm{zu}$ berücksichtigen, welche mit 2,7 $\mathrm{mm}$ $(k=1)$ einen nicht $\mathrm{zu}$ vernachlässigenden Beitrag zur Messunsicherheit leistet. Zusammenfassend zeigen die Ergebnisse an der kleinen Verzahnung das Potential des modellbasierten Multi-Distanz-Messansatzes zur Erfassung zahnindividueller Formparameter und bieten eine vielversprechende Grundlage für die zukünftige Anwendung an Großverzahnungen.

\section{Zusammenfassung}

In diesem Artikel wird die Eignung eines neuartigen modellbasierten Multi-Distanz-Messansatzes nach [11] zur Messung zahnindividueller Formparameter am Beispiel von Geradverzahnungen mit einer Messunsicherheit $<10 \mu \mathrm{m}(k=2)$ vorgestellt. Durch die Kombination eines optischen konfokal-chromatischen Abstandssensors mit einem Drehtisch kann die Oberfläche aller Zähne erfasst und anschließend modellbasiert ausgewertet werden. Zur Reduzierung von Unsicherheiten in der Messanordnung wird das Multi-Distanz-Messsystem mittels eines Kalibrierzahnrades kalibriert, sodass sich systematische Messabweichungen bei der Grundkreisradiusberechnung korrigieren lassen. Die Eignung zur Erfassung zahnindividueller Formparameter wird zunächst an kleinen Verzahnungen veranschaulicht.

Die experimentellen Untersuchungen zeigen, dass systematische Messabweichungen korrigiert werden und die Multi-Distanz-Messungen im Rahmen der erweiterten Messunsicherheit mit den Referenzmessungen übereinstimmen. Das Ziel, eine Messunsicherheit $<10 \mu \mathrm{m}(k=2)$ zu erreichen, ist erfüllt und demonstriert das Potential des Multi-Distanz-Messansatzes zur Erfassung von zahnindividuellen Formparametern. Die zukünftigen Forschungsaktivitäten konzentrieren sich daher auf die Skalierbarkeit des Multi-Distanz-Ansatzes, um entsprechende geometrische Messungen von Großverzahnungen zu validieren.

Danksagung: Die Autoren bedanken sich herzlich bei der Deutschen Forschungsgemeinschaft (DFG) für die Förderung des Forschungsprojektes (FI 1989/2-1).

\section{Literatur}

[1] F. Balzer, N. Steffens, M. Stein und K. Kniel. Traceable measurement of large gears with micron accuracy: a mandatory basis for reliable wind energy systems. In Engineering for a Changing World: Proceedings; 59th IWK, IImenau Scientific Colloquium, Band 59, Technische Universität Ilmenau, September 11-15, 2017.

[2] Y.-C. Chen und J.-Y. Chen. Optical inspection system for gear tooth surfaces using a projection moiré method. Sensors, 19(6):1450, 2019. 10.3390/s19061450.

[3] DIN EN ISO 15530-3. Geometrical product specifications (GPS) - Coordinate measuring machines (CMM): Technique for determining the uncertainty of measurement - Part 3: Use of calibrated workpieces or measurement standards (ISO 15530-3:2011), Sept. 2018.

[4] DIN ISO 1328-1. Cylindrical gears - ISO system of flank tolerance classification - Part 1: Definitions and allowable values of deviations relevant to flanks of gear teeth (ISO 1328-1:2013), Mar. 2018.

[5] F. Franceschini, M. Galetto, D. Maisano und L. Mastrogiacomo. Large-scale dimensional metrology (Isdm): from tapes and theodolites to multi-sensor systems. International Journal of Precision Engineering and Manufacturing, 15(8): 1739-1758, 2014. 10.1007/s12541-014-0527-2.

[6] G. Goch. Gear metrology. CIRP Annals, 52(2):659 - 695, 2003. 10.1016/S0007-8506(07)60209-1.

[7] A. Günther, J. Peters und G. Goch. Flächenhafte numerische beschreibung, ausrichtung und auswertung von zylinderrädern (3d-surface-like numerical description, alignment, and evaluation of involute cylindrical gears). tm - Technisches Messen Plattform für Methoden, Systeme und Anwendungen der Messtechnik, 68(4/2001):160, 2001.

[8] X. Guo, Z. Shi, B. Yu, B. Zhao, K. Li und Y. Sun. 3d measurement of gears based on a line structured light sensor. Precision Engineering, 61:160 - 169, 2020. 10.1016/j. precisioneng.2019.10.013.

[9] H. Linke, J. Börner und R. Heß. 8 - ensuring the accuracy of cylindrical gears. In H. Linke, J. Börner und R. Heß, editors, Cylindrical Gears, S. 554 - 589. Hanser, 2016. ISBN 978-1-56990-489-3. 10.3139/9781569904909.008.

[10] G. N. Peggs, P. G. Maropoulos, E. B. Hughes, A. B. Forbes, S. Robson, M. Ziebart und B. Muralikrishnan. Recent developments in large-scale dimensional metrology. Proceedings of the Institution of Mechanical Engineers, Part B: Journal of Engineering Manufacture, 223(6):571-595, 2009. 10.1243/09544054JEM1284.

[11] M. Pillarz, A. von Freyberg und A. Fischer. Gear shape parameter measurement using a model-based scanning multidistance measurement approach. Sensors, 20(14):3910 (16 pp.), 2020. 10.3390/s20143910.

[12] M. Pillarz, A. von Freyberg, D. Stöbener und A. Fischer. Gear shape measurement potential of laser triangulation and confocal-chromatic distance sensors. Sensors, 21(3):937(22 pp.), 2021. 10.3390/s21030937.

[13] D. Stöbener, A. von Freyberg, M. Fuhrmann und G. Goch. Areal parameters for the characterisation of gear distortions. Materia/wissenschaft und Werkstofftechnik, 43(1-2):120-124, 2012. 10.1002/mawe.201100898. 